\title{
Accretion Disc Thermal Reverberation in the Lamp Post Geometry ${ }^{\dagger}$
}

\author{
Iossif E. Papadakis ${ }^{1,2,3, *}$, Elias S. Kammoun ${ }^{4}$ and Michal Dovčiak ${ }^{5}$ \\ 1 Department of Physics and Institute of Theoretical and Computational Physics, University of Crete, \\ 71003 Heraklion, Greece \\ 2 Foundation for Research and Technology-Hellas, IESL, Voutes, 71110 Heraklion, Greece \\ 3 Institute of Astrophysics, FORTH, GR-71110 Heraklion, Greece \\ 4 Department of Astronomy, University of Michigan, 1085 South University Avenue, \\ Ann Arbor, MI 48109-1107, USA; ekammoun@umich.edu \\ 5 Astronomical Institute of the Academy of Sciences, Boční II 1401, CZ-14100 Prague, Czech Republic; \\ michal.dovciak@asu.cas.cz \\ * Correspondence: jhep@physics.uoc.gr \\ + Presented at the meeting Recent Progress in Relativistic Astrophysics, Shanghai, China, 6-8 May 2019.
}

Published: 18 October 2019

\begin{abstract}
We present results regarding the disc response functions and the corresponding time lags assuming a standard Novikov-Thorne accretion disc illuminated by a point-like X-ray source. We took into account all relativistic effects in the light propagation from the $\mathrm{X}$-ray source to the disc and then to the observer, and we computed the disc reflection, accounting for its radial ionization profile. Our results suggest that the thermal reverberation effects should be stronger in sources with large X-ray source height and low accretion rate. We found that time lags increase with height and accretion rate. The amplitude of the observed time lags as a function of wavelength (in NGC 5548) is consistent with the model predictions. It is not necessary for the disc to be too hot, it may be that the $\mathrm{X}$-ray source is located further from the disc.
\end{abstract}

Keywords: galaxies: active; galaxies: Seyfert; galaxies: individual (NGC 5548); X-rays: general

\section{Introduction}

Active galactic nuclei (AGN) are thought to be powered by accretion of matter onto a supermassive black hole (BH, with a mass $M_{\mathrm{BH}} \sim 10^{6-9} \mathrm{M}_{\odot}$ ) in the form of an optically-thick, geometrically-thin disc [1,2]. The disc emits a multi-temperature blackbody (BB) spectrum peaking in the optical/ultraviolet (UV) range. The temperature of the disc decreases with radius as $T(r) \propto r^{-3 / 4}$. A fraction of the disc photons are then Compton up-scattered by a medium of hot electrons in the vicinity of the $\mathrm{BH}$, the so-called 'X-ray corona'. If the X-ray source is small in size, if it is located above the $\mathrm{BH}$ at height $h$ (i.e., the 'lamp-post' geometry), and if the X-rays are emitted isotropically, then they will irradiate the accretion disc. Some of them will be absorbed, and will increase the disc's temperature. As a result, the disc's UV/optical emission will be enhanced.

Most of the thermally reprocessed UV photons are expected to emerge from the hot inner regions, while the optical photons are expected to be emitted by the outer regions. Consequently, if the X-rays are variable, we expect the part of the UV/optical emission which is due to X-ray absorption to also vary with delays increasing with wavelength.

We present results from a recent study [3] of the time lags vs. wavelength relation (hereafter "lag-spectrum") in the context of the lamp-post geometry. We investigated the effects of the X-ray source height and the accretion rate on the disc response functions and the lag spectra. We applied 
the model to NGC 5548 data from a recent monitoring campaign [4], and we found that a standard disc, with a low accretion rate ( $\sim 0.005-0.01$ of the Eddington limit) is in agreement with the observed $\mathrm{UV} /$ optical lags in this source, as long as the X-ray source height is larger than $40 \mathrm{r}_{\mathrm{g}}$.

\section{Model Setup}

We considered a Keplerian, geometrically-thin and optically-thick accretion disc, around a BH of mass $M_{\mathrm{BH}}$ and accretion rate $\dot{m}$. The disc is co-rotating with the $\mathrm{BH}$ and its temperature profile follows the Novikov-Thorne model prediction, with a color temperature correction factor of 2.4. The disc extends from the innermost stable circular orbit (ISCO) at radius $r_{\mathrm{ISCO}}$, up to an outer radius of $r_{\text {out }}=10^{4} \mathrm{r}_{\mathrm{g}}$. We considered two extreme cases, one with $a^{*}=0\left(r_{\mathrm{ISCO}}=6 \mathrm{r}_{\mathrm{g}}\right)$ and the other with $a^{*}=1\left(r_{\mathrm{ISCO}}=1 \mathrm{r}_{\mathrm{g}}\right)$.

We also assumed a point-like X-ray source located at a height $(h)$ on the rotational axis of the $\mathrm{BH}$ (i.e., the lamp-post geometry). X-rays are emitted isotropically (in the rest frame of the lamp-post) with an intrinsic spectrum $F_{X}(t)=N(t) E^{-\Gamma} \exp \left(-E / E_{C}\right)$, and illuminate the disc. Part of the X-ray flux is reprocessed and re-emitted in X-rays (this is the 'disc reflection component') and part of it is absorbed. We assumed that the $\mathrm{X}$-ray source is variable in normalization only.

The main objective of our work is to compute the disc extra emission (due to X-ray absorption), as a function of time, in the case of when the disc is illuminated by an X-ray flash at time $t_{0}$. To this end, we did the following:

1. Given the observed 2-10 keV luminosity, we estimated the intrinsic luminosity and the incident flux on each disc radius, as a function of time (in the observer's frame), taking into account all the relativistic effects in the propagation of light from the primary source to the disc.

2. Given the incident $X$-ray flux on the disc at each radius, the model estimates the radial ionization profile of the accretion disc assuming a constant disc density. The disc reflection spectrum was computed using the XILLVERD tables for the reflection spectrum from ionized material [5], by integrating them from $0.1 \mathrm{keV}$ to infinity. The XILLVERD table model provides a new and complete library of synthetic spectra for the emission that is reflected from an X-ray illuminated accretion disc, taking into account its ionization state.

3. We estimated the X-ray flux that each disc element absorbs. We add to that the intrinsic flux of the disc, and we computed the new disc temperature.

4. We identified all of the disc elements (in radius, $r$, and azimuth, $\varphi$ ) that a distant observer will "see" to be illuminated at the same time, and we computed the flux that the observer receives, at a time $\tau_{\mathrm{obs}}$, from all these elements in a given waveband (taking into account all relativistic effects in the propagation of light from the disc to the observer).

5. The disc response function in this waveband is the difference between this flux and the flux of the same disc elements, in the same band pass, when their temperature is equal to the Novikov-Thorne disc. Defined in this way, the response function shows the "extra" flux that is emitted by the disc, at each time $\tau_{\text {obs }}$ (in the observer's frame), due to the heating caused by the absorption of the incident $\mathrm{X}$-rays.

\section{The Disc Response Functions}

To compute the disc response we chose model parameter values that are applicable for NGC 5548. In particular, we assumed $M_{\mathrm{BH}}=5 \times 10^{7} \mathrm{M}_{\odot}$ [6], inclination of $40^{\circ}, L_{X} / L_{\mathrm{Edd}}=0.0034$, a high-energy cutoff of $300 \mathrm{keV}$ in the X-ray spectrum, and a luminosity distance of $75 \mathrm{Mpc}$. We computed the disc response functions, $\Psi(t)$, in most of the wavebands presented by [4]. We considered eight values for the lamp-post height $\left[h\left(\mathrm{r}_{\mathrm{g}}\right)=2.5,5,10,20,40,60,80,100\right]$ and eight values of the accretion rate $\left[\dot{m} / \dot{m}_{\text {Edd }}(\%)=0.25,0.5,0.75,1,2.5,5,7.5,10\right]$. We assumed a disc density $n_{\mathrm{H}}=10^{17} \mathrm{~cm}^{-3}$, and BH spins $a^{*}=0$ and 1 . 
The left panel of Figure 1 shows the response functions for all the values of $\dot{m}$, for $a^{*}=0$ and 1 , in the HST $\lambda 1158$ and the $I$ bands (the shortest and longest wavelengths, respectively), for $h=10 \mathrm{rg}_{\mathrm{g}}$. The main characteristics of the response functions are as follows:

1. The responses for all accretion rates (in all bands) start rising at the same time, and

2. $\Psi(t)$ increases in amplitude and gets narrower as the accretion rate decreases.

The right panel of Figure 1 shows the response functions for all the values of $h$, for spins 0 and 1 , $\dot{m} / \dot{m}_{\mathrm{Edd}}=1 \%$ and the HST $\lambda 1158$ and the $I$ bands. The main results are as follows:

1. The larger the height of the lamp-post the later the response function starts, and the longer it lasts.

2. The amplitude of the response functions increases with height.

Finally, the response functions at low spin are broader and have lower amplitudes than the ones for $a^{*}=1$.
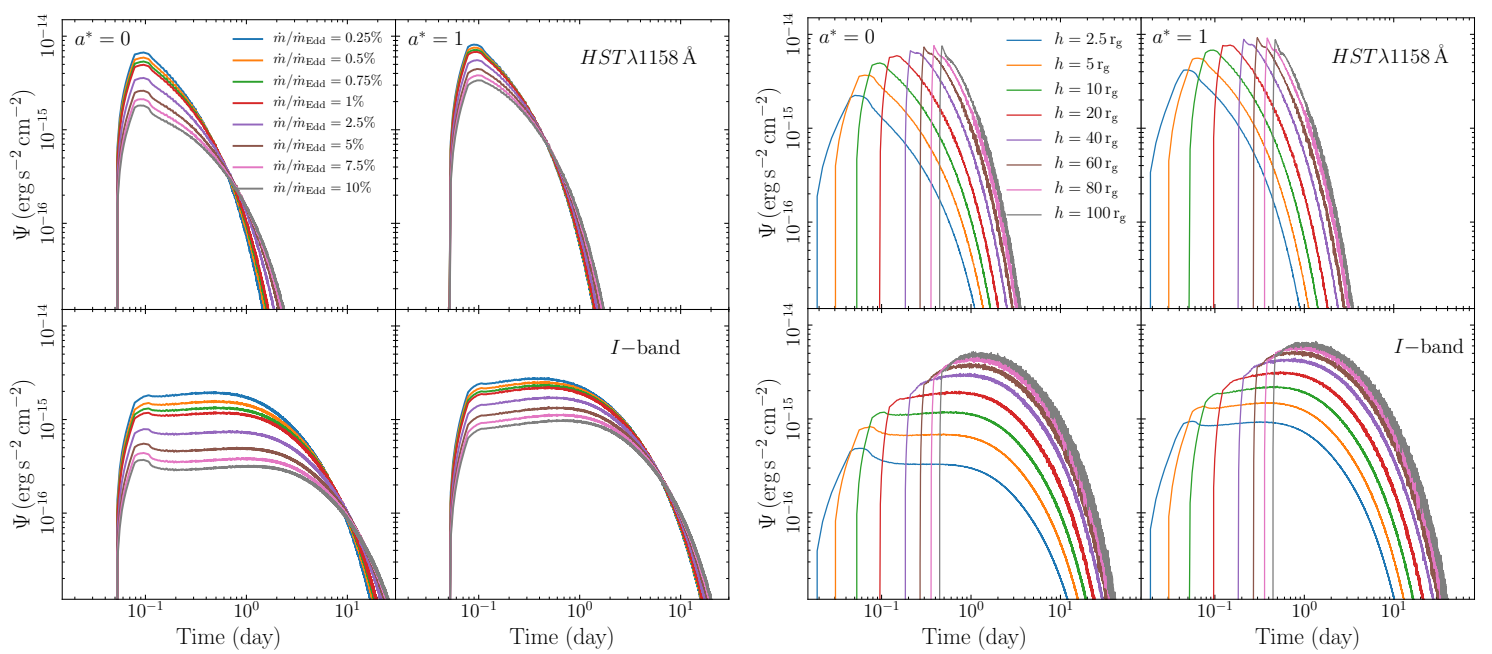

Figure 1. (Left panel) Response functions for the various $\dot{m}$ values we considered $\left(h=10 \mathrm{r}_{\mathrm{g}}\right.$ ). (Right panel) the same for the various $h$ values we considered ( $\dot{m}=0.01 \dot{m}_{\text {Edd }}$ ). Left and right columns correspond to $a^{*}=0$ and 1 , respectively. Top and bottom rows show the responses for the HST $\lambda 1158$ and the $I$-band, respectively.

\section{The Time Lags in NGC 5548}

We estimated the centroid time delay of the transfer functions at a given wavelength $(\lambda)$ as follows,

$$
\langle\tau(\lambda)\rangle=\frac{\int \tau \Psi(\tau, \lambda) \mathrm{d} \tau}{\int \Psi(\tau, \lambda) \mathrm{d} \tau}
$$

It is this mean time lag that we can compare with the observed time lags between X-rays and UV/optical light curves. We estimated the $\chi^{2}$ between the model lag-spectra (for all $\dot{m}$ and heights; 64 in total for each spin) and the time lags between the X-ray and the UV/optical light curves in NGC 5548 (excluding the $U$-band point from the fit due to the possibility of an additional delay caused by the broad line region to the observed lag in this band). We obtained statistically accepted fits $\left(\chi_{\min }^{2} / d o f=10.8 / 7\right.$ and 10.7/7; $p$-value $=0.15$ and 0.16$)$ for $a^{*}=0$ and $a^{*}=1$, respectively. The filled points in Figure 2 show the observed delays in NGC 5548 (with respect to the shortest wavelength FUV band) and the solid (dashed) lines in the same figure indicate the best-fit model to the observed lag spectrum for $a^{*}=0\left(a^{*}=1\right)$. 


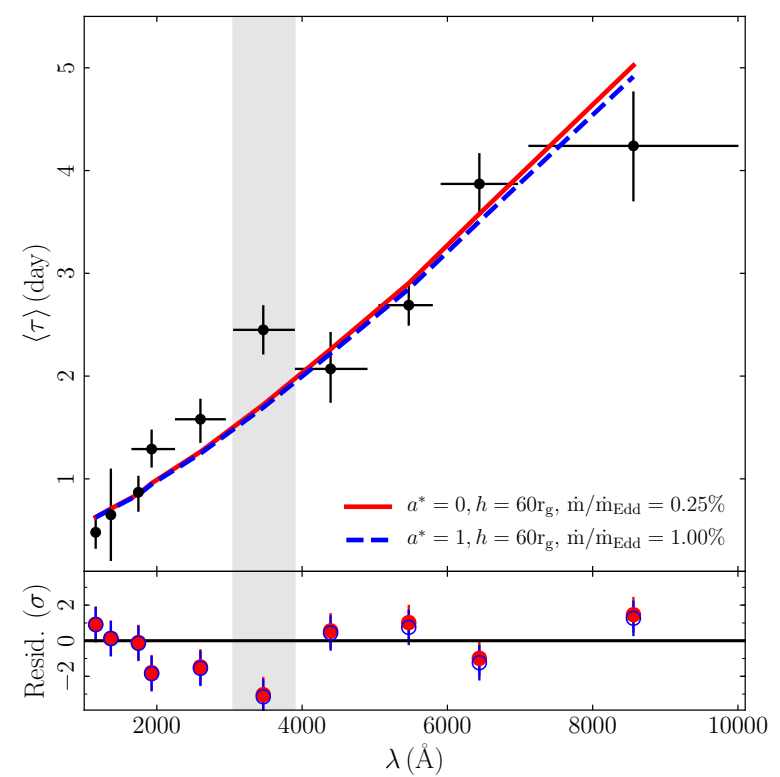

Figure 2. Best-fit models for $a^{*}=0$ (solid line) and $a^{*}=1$ (dashed line) obtained by fitting the observed time lags, excluding the measurement in the $U$-band (shaded region).

The best-fit $h$ and $\dot{m}$ values are $\left(60 \mathrm{r}_{\mathrm{g}}, 0.25 \% ; a^{*}=0\right)$ and $\left(60 \mathrm{r}_{\mathrm{g}}, 1 \% ; \mathrm{a}^{*}=1\right)$. The best-fit $\dot{m}$ in the $a^{*}=0$ case is equal to the lowest value we considered, with a 3- $\sigma$ upper limit of $2.5 \%$. The $1-\sigma$ confidence region of $\dot{m}$ for the maximally rotating BH is $0.25-2.5 \%$. The best-fit height of the X-ray source are identical in both cases, with the 3- $\sigma$ confidence region being $\left(40-80 r_{g}\right)$ and $\left(20-80 r_{g}\right)$ for $a^{*}=0$ and $a^{*}=1$, respectively.

\section{Conclusions}

We present results from a recent study, where we calculated the disc response functions in various wavebands when the disc is illuminated by $\mathrm{X}$-rays assuming a lamp-post geometry. We considered all relativistic effects in the light propagation from the $\mathrm{X}$-ray source to the disc and from the disc to the observer. We also accounted for the disc X-ray reflection by computing the disc ionization at each radius. Our main results are as follows:

- The delays between X-rays and optical/UV bands increase with increasing source height and increasing accretion rate.

- The disc response in all UV/optical bands increases when the source height increases and the accretion rate decreases. Therefore, we do not expect a strong thermal reverberation signal in objects with high accretion rate and strong $\mathrm{X}$-ray reflection signatures like, for example, the $\mathrm{X}$-ray bright narrow-line Seyfert-1 galaxies.

Using reasonable values for the model parameters (i.e., BH mass, inclination, X-ray spectral slope, and mean flux) we explained the observed time-lag spectrum in NGC 5548. The best-fit results indicate a source height larger than 20 or $40 \mathrm{r}_{\mathrm{g}}$ (3- $\sigma$ limit for $a^{*}=1$ or 0 , respectively). This is in agreement with [7] who inferred a height of the X-ray source in NGC $5548 \sim 100 \mathrm{rg}_{\mathrm{g}}$.

As for the accretion rate, the best-fit values indicate rates which are $\sim 1$ per cent of the Eddington limit. According to [4], the mean source flux at $5100 \AA$ is $\sim 4.6 \times 10^{-11} \mathrm{erg} \mathrm{s}^{-1} \mathrm{~cm}^{-2}$. Assuming a bolometric correction factor of $7.8 \pm 1.7$ [8], this implies a bolometric luminosity of $(2.5 \pm 0.5) \times 10^{44} \mathrm{erg} \mathrm{s}^{-1}$, which is $0.034 \pm 0.008$ of the Eddington luminosity limit for $M_{\mathrm{BH}}=$ $5.7 \times 10^{7} \mathrm{M}_{\odot}$. This value is at odds with the $\dot{m}$ estimate for $a^{*}=0$ (the $3 \sigma$ upper limit is just 0.025 ) but entirely consistent with the $\dot{m}$ estimate considering a standard accretion disc around a maximally rotating $\mathrm{BH}$. 
Author Contributions: All authors contributed equally to this work.

Funding: M.D. thanks MEYS of the Czech Republic for the support through the 18-00533S research project and his home institution, ASU, supported by the project RVO:67985815.

Acknowledgments: IP would like to thank ASU for hospitality.

Conflicts of Interest: The authors declare no conflict of interest.

\section{References}

1. Novikov, I.D.; Thorne, K.S. Astrophysics of black holes. In Black Holes (Les Astres Occlus); Dewitt, C., Dewitt, B.S., Eds.; ARI: Heidelberg, Germany, 1973; pp. 343-450.

2. Shakura, N.I.; Sunyaev, R.A. Black holes in binary systems. Observational appearance. Astron. Astrophys. 1973, 24, 337-355.

3. Kammoun, E.S.; Papadakis, I.E.; Dovčiak, M. A hard look at thermal reverberation and optical/ultraviolet lags in NGC 5548. Astrophys. J. 2019, 879, L24.

4. Fausnaugh, M.M.; Denney, K.D.; Barth, A.J.; Bentz, M.C.; Bottorff, M.C.; Carini, M.T.; Croxall, K.V.; de Rosa, G.; Goad, M.R.; Horne, K.; et al. Space telescope and optical reverberation mapping project. III. Optical continuum emission and broadband time delays in NGC 5548. Astrophys. J. 2016, 821, 56.

5. García, J.A.; Fabian, A.C.; Kallman, T.R.; Dauser, T.; Parker, M.; Mcclintock, J.E.; Steiner, J.F.; Wilms, J. The effects of high density on the X-ray spectrum reflected from accretion discs around black holes. Mon. Not. R. Astron. Soc. 2016, 462, 751.

6. Bentz, M.C.; Katz, S. The AGN black hole mass database. Publ. Astron. Soc. Pac. 2015, 127, 67.

7. Brenneman, L.W.; Elvis, M.; Krongold, Y.; Liu, Y.; Mathur, S. Ngc 5548: Lack of a broad fe k $\alpha$ line and constraints on the location of the hard X-ray source. Astrophys. J. 2012, 744, 13.

8. Krawczyk, C.M.; Richards, G.T.; Mehta, S.S.; Vogeley, M.S.; Gallagher, S.C.; Leighly, K.M.; Ross, N.P.; Schneider, D.P. Mean spectral energy distributions and bolometric corrections for luminous quasars. Astrophys. J. Suppl. Ser. 2013, 206, 4.

(C) 2019 by the authors. Licensee MDPI, Basel, Switzerland. This article is an open access article distributed under the terms and conditions of the Creative Commons Attribution (CC BY) license (http:/ / creativecommons.org/licenses/by/4.0/). 